\title{
As plantas medicinais, a Política Nacional de Práticas Integrativas e Complementares do SUS e Projeto Farmácia Verde em São Lourenço, MG
}

\author{
Rodrigo Framil de Souza CUNHA ${ }^{1}$, Maria Alice Fernandes Corrêa MENDONÇA ${ }^{2 *}$, Eliana AlCÂNTRA ${ }^{3}$
}

1 Universidade Vale do Rio Verde, Três Corações

2 Docente do departamento de Economia Rural, Universidade Federal de Viçosa (UFV)

3 Programa de Pós-Graduação em Sustentabilidade e Recursos Hídricos, Universidade Vale do Rio Verde, Três Corações

* Autora para correspondência - maria.alice.fcm@gmail.com

Recebido em 14 de janeiro de 2019. Aceito em 21 de julho de 2019. Publicado em 31 de julho de 2019.

Resumo - A implantação da PNPIC-SUS reforça a importância de se identificar e reconhecer o conhecimento popular sobre as plantas medicinais. Assim objetivou-se com esse trabalho mapear o cultivo e uso de plantas medicinais entre os usuários do Sistema Único de Saúde (SUS), cadastrados no Programa Saúde da Família no município de São Lourenço, Minas Gerais. Como método de pesquisa, realizaram-se, no período de setembro e outubro de 2018, entrevistas com 50 participantes. Ainda, foram coletadas mudas, ramos com folhas e flores das plantas cultivadas como amostras, as quais foram devidamente identificadas com seus nomes científicos e famílias. Como resultado, revelou-se um mapa etnobotânico da área da UBS Vila Nova em São Lourenço, com o levantamento das plantas medicinais mais utilizadas para tratamentos diversos. Catalogaram-se 180 amostras de 46 espécies distintas. Conclui-se que as plantas medicinais para fins terapêuticos, ou por hábito de consumo, são muito utilizadas pela população cadastrada no Programa Saúde da Família na UBS do bairro Vila Nova na cidade de São Lourenço, sendo que a maioria dos usuários cultiva suas plantas devido a suas origens rurais e seu conhecimento empírico passado por seus ancestrais. Os dados desta pesquisa podem contribuir para o reconhecimento do saber popular sobre as plantas medicinais. Além disso, reitera-se a importância social, econômica e cultural das políticas públicas ao se destacar também os desafios para o pleno conhecimento e usufruto das mesmas pela população.

Palavras-Chave: Plantas medicinais; Terapias Complementares; Etnobotânica; Saúde Pública; Políticas Públicas

The medicinal herbs, National Policy on Integrative and Complementary Practices of the SUS and the Green Pharmacy Project in São Lourenço, MG

\begin{abstract}
Aвstract - The implementation of the National Policy on Integrative and Complementary Practices of the SUS (PNIPIC-SUS), reinforces the importance on identification and recognition of popular knowledge of medical herbs. The central objective of this paper was to map and characterize the grow and use of medical herbs between users of the Brazilian health system (SUS), registered on the Family Heathy Program, at São Lorenço, Minas Gerais. As research method for data collection, 50 interviews with participants were taken between September and October of 2018. Also, seedlings, branches with leaves and flowers of the cultivated plants were collected as samples, which were properly identified with their scientific names and families. As result, we produced an ethnobotanic map, on the research area. On this map, we demonstrate the most used herbs, their main use for diverse treatments. We catalog 180 samples of 46 different species. We conclude that the herbs, used for therapeutic purpose, or by cultural custom, are widely used by SUS users, at the study locality in São Lourenço. The popular knowledge is commonly passed through generations. The data from this research may contribute to the recognition of popular knowledge about medicinal plants. Moreover, reiterates the social, economic and cultural importance of public policy to support local experiences.
\end{abstract}

Keywords: Medicinal herbs; Complementary therapies; Ethnobotanics; Public health; Public Policy. 
Las plantas medicinales, la Política Nacional de Prácticas Integrativas y Complementarias del SUS (Sistema brasileño de salud) y el Proyecto de Farmacia Verde en São Lourenço, Mg

Resumen - La implementación de una política pública especial llamada PNIPIC-SUS, refuerza la importancia de la identificación y el reconocimiento del saber popular de las plantas medicinales. El objetivo central de la investigación fue mapear el cultivo y el uso de plantas medicinales entre los usuarios del sistema brasileño de salud (SUS), registrado en el programa salud de la familia, en São Lourenço, Minas Gerais. Como método de investigación se encuestó a 50 participantes en el periodo de septiembre y octubre de 2018. También, los investigadores recolectaron plántulas, ramas con hojas y flores de plantas cultivadas como muestras para la debida identificación con sus nombres científicos y familias. Como resultado, se elaboró un mapa etnobotánico del área de investigación. En este mapa, enseñamos las plantas más utilizadas y su uso principal para diversos tratamientos. Se catalogaron 180 muestras de 46 especies distintas. Se concluye que las plantas utilizadas con fines terapéuticos, o por las costumbres culturales, son ampliamente utilizadas por los usuarios del programa de salud de la familia en el área de investigación, São Lourenço. El conocimiento popular es comúnmente pasado a través de generaciones. Los datos generados por esta investigación pueden contribuir para el reconocimiento del saber popular acerca de las plantas medicinales. Además, se reitera la importancia social, económica y cultural de las políticas públicas al destacar los retos para el total conocimiento y usufructo de la población.

Palabras Clave: Plantas medicinales; Terapias complementarias; Etnobotánico; Salud pública; Política pública

\section{INTRODUÇÃO}

Em 1988, com a publicação das resoluções da Comissão Interministerial de Planejamento e Coordenação (CIPLAN) foram criados inúmeros projetos e programas de fitoterapia na rede pública de saúde, possibilitando a criação de práticas e rotinas de atendimentos nos municípios, por meio das Unidades de Atendimento Básico de Saúde (Brasil 2015). Em 2006, a implementação da Política Nacional de Práticas Integrativas e Complementares no Sistema Único de Saúde (PNPIC-SUS) visou estimular ações para a promoção da saúde dos usuários do Sistema Único de Saúde (SUS) através de práticas terapêuticas alternativas à medicina ocidental convencional, como: acupuntura, homeopatia, plantas medicinais e fitoterapia (Trindade et al. 2008).

A PNPIC-SUS partiu de uma demanda da Organização Mundial da Saúde (OMS), a qual “(...) estimula, desde o final da década de 70, a implantação da chamada Medicina Tradicional ou Medicina Complementar e Alternativa nos Sistemas de Saúde" (Sousa et al. 2012, p. 2143). Além de trazer consigo um resgate e valorização de costumes e culturas ancestrais, a PNPIC-SUS contribuiu para o fortalecimento dos princípios fundamentais do sistema de saúde público, bem como para a integralidade da atenção à saúde, estabelecendo normas e complementações dessas práticas terapêuticas na rede de saúde pública.

Em conformidade com as políticas nacionais, em dezembro de 2008 foi aprovado o Programa Nacional de Plantas Medicinais e Fitoterápicos com representantes de diversas partes do território brasileiro, sendo esse responsável por definir responsabilidades e prazos para o cumprimento das ações no âmbito da produção e atendimento às demandas práticas da PNPIC-SUS (Brasil 2012).

Nesse sentido, a implantação da PNPIC-SUS no município de São Lourenço, estado de Minas Gerais, especialmente a partir do Projeto Farmácia Verde, reforçou a importância de se identificar e valorizar o conhecimento popular local sobre as plantas medicinais. Não apenas no sentido de aplicação local desse conhecimento, mas também no sentido de aproximar o sistema público de saúde da população usuária e, para além disso, de incorporar a esse sistema, seus valores de saúde, suas práticas e porque não, seus quintais medicinais. Desse modo, três perguntas moveram o interesse para o desenvolvimento da pesquisa de campo que resultou neste trabalho, são elas: a) como aproximar o SUS e os cultivadores de plantas medicinais no município de São Lourenço?; b) quem cultiva 
as plantas medicinais, conhece o Projeto Farmácia Verde da Unidade Básica de Atendimento Vila Nova? e; c) O Projeto Farmácia Verde conhece os cultivadores e os modos de cultivo e usos da população local?

No entendimento dos pesquisadores, essas questões constituem uma premissa fundamental para a ética na pesquisa etnobotânica. Se interessa à Farmácia Verde compreender melhor o universo dos usuários e produtores de plantas medicinais locais, pensa-se que a pesquisa etnobotânica é o melhor caminho para isso. Ademais, o SUS e a população local cultivadora das plantas medicinais precisam se (re)conhecer para o sucesso da política pública. Nesse caso, especialmente, por meio do papel desempenhado pelos agentes comunitários de saúde do SUS.

Assim objetivou-se com esse trabalho mapear e catalogar o cultivo e uso de plantas medicinais pelos usuários do Sistema Único de Saúde (SUS) em uma região no município de São Lourenço, Minas Gerais. Assim como, analisar a integração da comunidade usuária e o Projeto Farmácia Verde, da Secretaria Municipal de Saúde por meio de pesquisa qualitativa através de entrevistas estruturadas.

\section{Material e MÉtodos}

Área de estudo

A cidade de São Lourenço está localizada na região sul de Minas Gerais, faz parte do Circuito das Águas e da Serra da Mantiqueira. As coordenadas geográficas da cidade são de latitude: $22^{\circ} 06$ '59"S, longitude: $45^{\circ} 03^{\prime} 16^{\prime}$ W e altitude de $874 \mathrm{~m}$. A microrregião onde se localiza é composta por 16 municípios com população estimada em 217.532 habitantes com área total de $3.831 .155 \mathrm{Km} 2$ de clima tropical de altitude e vegetação de mata atlântica (IBGE 2013). Sua população é estimada em 44.781 habitantes (IBGE 2015) e sua economia gira em torno basicamente do turismo (águas minerais), comércio e setores de serviços.

No contexto político institucional que surge o campo da pesquisa resulta o presente trabalho. A pesquisa foi desenvolvida no âmbito do Projeto Farmácia Verde instituída desde 23 de outubro de 2014 no município de São Lourenço, MG, que refere-se ao Programa Farmácia Viva do Ministério da Saúde (Portaria número 886 de 20 de abril de 2010) desenvolvido pela Universidade Federal do Ceará com a finalidade oferecer suporte à população no que tange o acesso e garantia à saúde pública, por meio da utilização da flora regional (BRASIL 2012).

A Farmácia Verde atualmente trabalha com quatro espécies de plantas medicinais, sendo elas: Calendula officinalis (calêndula), Aloe vera (babosa), Mikania glomerata / M. laevigata (guaco) e Maytenus ilicifolia (espinheira santa). Como o projeto não conta com a implantação de um cultivo permanente, a aquisição das plantas é feita através, principalmente, de produtores locais que seguem as normas para essa finalidade e doações de produtores orgânicos da região, respeitando todos os critérios fitossanitários, o que justifica ainda mais a aproximação colaborativa e ética entre as partes envolvidas.

\section{A Unidade Básica de Saúde Vila Nova}

A Unidade Básica de Saúde (UBS) localizada no bairro Vila Nova atende cerca de 400 pessoas mensalmente através de consultas marcadas na agenda da unidade. Essas, fazem parte do cadastro do Programa Saúde da Família (PSF) contendo um total aproximado de 1100 famílias (em torno de 3500 pessoas). As famílias atendidas são residentes dos bairros Jardim Paraíso, Residencial Rio Verde, Parque Olímpico e Centro. Parte dessas famílias é de origem rural e é de costume o hábito do cultivo de hortas e pomares nas residências. A maior parte do bairro é constituída por casas, e seus moradores trabalham no setor comercial, serviços e turístico. 
A equipe da UBS Vila Nova é composta por uma enfermeira chefe, um clínico geral, dois técnicos de enfermagem, dois dentistas, um auxiliar de dentista, um auxiliar de serviços gerais, seis agentes de saúde, dois técnicos administrativos e uma psicóloga. A UBS atua em conjunto com o Núcleo de Apoio à Saúde da Família (NASF), o grupo Farmácia Verde, e o Centro de atenção Psicossocial (CAPS). Como parte das atividades das equipes são realizadas visitas domiciliares compartilhadas com outros profissionais para pessoas que são acamadas e domiciliadas a fim de ampliar o acesso do atendimento pela unidade.

A unidade é focada na prevenção e educação junto à comunidade em relação às práticas de saúde e hábitos de vida mais saudáveis. Para isso são formados grupos de hipertensos, diabéticos e saúde mental dentre outros, para a discussão construtiva de casos específicos.

Farmacinha é o nome popular para a Farmácia básica de São Lourenço onde a população pode retirar seus medicamentos sem custos através de uma receita emitida pelo médico dos programas de saúde da família nas unidades básicas da cidade. A Farmácia Verde funciona na mesma estrutura física que a básica que a maioria da população já conhece.

\section{Coleta e Análise de Dados}

A pesquisa de campo foi realizada com o apoio da Secretaria Municipal de Saúde, da UBS Vila Nova e do Projeto Farmácia Verde, com a aprovação do Comitê de Ética em Pesquisa com seres humanos da Universidade Vale do Rio Verde (CEP-UninCor), sob o número do parecer: 2.907.149. Todas as pessoas entrevistadas durante a condução da pesquisa assinaram um Termo de Consentimento Livre e Eslarecido (TCLE).

Inicialmente realizaram-se palestras e reuniões para explicação do conteúdo do trabalho juntamente com a equipe, durante o mês de agosto de 2018, previamente à coleta de dados. Nessas reuniões participaram o Núcleo de Apoio a Saúde da Família e a profissional farmacêutica fitoterapeuta responsável pela Farmácia Verde.

Segundo Albuquerque et al. (2014), as pesquisas em Etnoecologia exigem uma interdisciplinaridade teórica e metodológica, que transcendem as barreiras rígidas dos estudos qualitativos ou quantitativos e mesclam instrumentos das diversas ciências para estudar as relações entre os seres humanos e as plantas. Dito isso, a entrevista é um dos instrumentos mais utilizados para os estudos em Etnoecologia, devendo ser conduzidas nas casas e/ou nos territórios dos entrevistados, no caso desta pesquisa foram os quintais.

Para Albuquerque et al. (2014), em entrevistas estruturadas, as questões previamente estabelecidas são colocadas de forma igual para cada informante, independente de contatos anteriores com o público participante. Essa abordagem exige que os pesquisadores tenham o domínio das questões mais relevantes, compreendendo por inteiro o propósito das perguntas. Ainda, segundo os autores, as entrevistas estruturadas impõem limites ao entrevistado (ou respostas do entrevistado); no entanto, têm a vantagem de facilitar a codificação / categorização das respostas, permitindo uma produção mais rápida de materiais para análise.

No decurso do trabalho de campo foram amostrados 50 quintais, nos meses de setembro e outubro de 2018. Foram aplicados dois formulários de entrevistas estruturadas para 50 pessoas (uma por quintal). Os participantes foram selecionados a partir do cadastro no Programa Saúde da Família do SUS. Foi dada a preferência às pessoas que se sabia, ou que se tinha uma percepção inicial de que cultivavam sua própria planta medicinal e a preparavam de forma caseira, a partir da interação desse público com os agentes comunitários.

As entrevistas foram distribuídas para os agentes comunitários de saúde na Unidade Básica de Saúde do bairro Vila Nova, divididas para 6 agentes comunitários de 6 micro-áreas distintas da UBS Vila Nova. A participação dos agentes foi fundamental para a confiança e proximidade já pré-estabelecida com a população entrevistada conforme indicado nos métodos (Albuquerque et al. 2014). Todos os participantes responderam os dois formulários de entrevistas. Esses foram aplicados oralmente, pelos agentes comunitários, os quais preencheram as respostas e adicionaram informações conforme a necessidade. 
O primeiro formulário de entrevista teve por objetivo introduzir a temática das plantas medicinais, sendo feitas as seguintes perguntas: 1) tem o hábito de tomar chá ou usar plantas medicinais; 2) cultiva suas próprias plantas; 3) conhece o projeto Farmácia Verde; 4) se afirmativo, acha esse projeto importante para a saúde da população de São Lourenço.

O segundo formulário abordou o cultivo e a utilização caseira das plantas, com as seguintes perguntas: 1) o nome popular da planta; 2) local de cultivo; 3) forma de preparo; 4) parte utilizada do vegetal e 5) a finalidade terapêtica a que se destina o preparo.

A cada participante foi solicitada a permissão para a retirada de uma amostra das plantas que cultivavam. A amostra consistiu da mudas (plantas com sistema radicular, caule, ramos, folhas e flores) e quando não foi possível a retirada da muda, foram coletados ramos com folhas e/ou flores que foi imediatamente colocada para secagem para montagem da exsicata. As plantas domesticadas já conhecidas, bem como plantas silvestres, cujos nomes populares foram dados foram identificadas com base em literatura especializada (Lorenzi e Matos 2008) e com o auxílio da farmacêutica especialista em fitoterápicos da Farmácia Verde.

É importante destacar como aspecto metodológico central desta pesquisa o cuidado para que as pessoas ficassem à vontade para expressar seu conhecimento sobre as plantas medicinais e seus efeitos nas formas de administração que elas praticavam, possibilitando assim um contato mais íntimo e fidedigno com esse conhecimento popular.

Como última etapa da pesquisa, a partir de um mapa disponibilizado pela Secretaria de Saúde realizou-se o registro das espécies vegetais coletadas, com a identificação e número de ocorrências na região estudada por meio do registro da localização dos participantes da pesquisa. O mapa usado já era utilizado pelos funcionários da UBS, especialmente agentes comunitários. De forma a contribuir com o trabalho dos agentes, resolveu-se pela catalogação das espécies (cultivo e uso) neste mesmo mapa, e não produzir um novo. Destaca-se aqui, contudo, o reconhecimento dos pesquisadores quanto à importância da confecção de mapas a partir dos conhecimentos populares locais, como um aspecto metodológico mais apropriado aos princípios da etnoecologia (Albuquerque et al. 2014).

\section{RESUlTADOS E DISCUSSÃo}

\section{O cultivo das plantas e a integração com o Projeto Farmácia Verde}

Quarenta e uma pessoas (82\%) responderam cultivar e fazer uso de plantas medicinais. Curiosamente, no entanto, nove pessoas (18\%) relataram cultivar as plantas em suas residências, porém, não fazendo uso das mesmas. Dessas, a maioria afirmou ter o hábito de doar as plantas medicinais para vizinhos, parentes e amigos. Outras mencionaram que determinadas plantas nasceram espontaneamente em seus quintais e passaram a cuidar destas a partir de então. As plantas que mais apareceram, segundo relatos, de modo espontâneo foram a Tanchagem (Plantago major L.) e o Capim limão (Cymbopogon citratus (D.C.) Stapf).

Predominantemente, segundo os entrevistados, as plantas medicinais são cultivadas em hortas e quintais $(60 \%)$, muitas vezes junto a outras plantas alimentícias, como hortaliças e frutíferas. Algumas pessoas relataram que, por não possuírem espaço de horta, cultivam suas plantas medicinais em vasos, jardineiras, latas e diversos outros objetos que entraram na categoria vasos $(22 \%)$. Cinco dos entrevistados $(8 \%)$ cuidam de plantas que nasceram espontaneamente e seis $(10 \%)$ dentre essas pessoas cultivam em mais de um local. Esses dados podem indicar uma característica correspondente aos traços culturais dessa população advinda de áreas rurais. Em sintonia com a análise De Mello Botelho et al. (2014), provavelmente, o hábito de cultivo é um traço remanescente desse lugar rural, e permaneceu quando essas pessoas ou seus antepassados migraram para a cidade, mesmo na ausência de um espaço apropriado para a agricultura. 
Outro resultado interessante revelou que a maioria dos usuários do SUS entrevistados não conheciam o Projeto Farmácia Verde apesar dos esforços da administração municipal para a implementação da Política Nacional de Práticas Integrativas e Complementares (PORTARIA No- 971, DE 3 DE MAIO DE 2006), através das plantas medicinais e dos fitoterápicos, oferecidos sob prescrição médica na atenção básica. Das cinquenta pessoas que participaram da pesquisa, somente nove $(18 \%)$ conheciam o projeto. No momento da abordagem foi questionado o conhecimento sobre o projeto por outro nome ("projeto farmacinha") ou pela localidade e mesmo assim quarenta e uma pessoas $(82 \%)$ mostraram desconhecimento.

Segundo Souza (2006), são aspectos de eficiência de uma política pública, dentre outras questões, a capacidade de: implementação, execução e avaliação da política. A autora ainda considera que as políticas públicas são projetos de longo prazo, mas devem ser constantemente avaliadas para aprimoramento e correções. Logo, os resultados obtidos na presente pesquisa podem indicar necessidade de se aprimorar essa relação, ou integração, entre o Projeto Farmácia Verde e o público-alvo.

Após a explicação dos agentes comunitários sobre o funcionamento do projeto, o público entrevistado mostrou interesse, avaliando o projeto como sendo importante para a qualidade e manutenção da saúde da população. Quarenta e nove pessoas ( $98 \%$ ) responderam positivamente e apenas uma pessoa (2\%) negativamente. Algumas dessas pessoas confirmaram que conheceram o projeto através dessas atividades na UBS e ainda mencionaram algumas das boas práticas do uso adequado das plantas medicinais oferecidas nos grupos, tais como, a escolha das partes sadias das plantas, a dosagem adequada e a forma correta do preparo para cada tipo de planta. Essas informações, permitiram inferir que a interação (projeto - público-alvo) parece existir, dado as informações sobre preparo informada pelos entrevistados. Porém, o nível de integração pode não ser suficientemente marcante para que esse público conseguisse identificar o projeto pela primeira pergunta. Nesse sentido, uma análise possível, ainda a partir da revisão de Souza (2006), é de que a força da interação entre a política pública e o público-alvo ocorre por meio do compartilhamento de ações, identidades e valores, construídos e reconstruídos repetidamente e continuamente ao longo do tempo. Contudo, para o sucesso dessa construção, é necessário avaliar sempre sua evolução.

As plantas e seus usos

No total das 50 casas de famílias visitadas foram retiradas 180 amostras de plantas de 46 espécies distintas, variando a quantidade de amostras de cada família. Dessas, foram destacadas 13 espécies de plantas medicinais mais cultivadas recolhidas na região que atende a UBS (Unidade Básica de Saúde) Vila Nova (Quadro 1).

Dentre todas as plantas cultivadas, a hortelã (Mentha villosa Huds.) foi a que se destacou mais com 28 amostras recolhidas. Seguida do alecrim (Rosmarinus officinallis L.) com 14 amostras, da erva cidreira (Melissa officinallis L.) com 12, juntamente com o bálsamo (Sedum dendroideum Moc.), a tanchagem (Plantago major L.), o funcho (Foeniculum vulgare Mill.), boldo brasileiro (Plectranthus barbatus A.), arruda (Ruta gaveolens L.) todas com 6 amostras, manjericão (Ocimum basillicum L.) e salsinha (Petroselinum crispum Mill.) ambas com 5 amostras coletadas e a caninha do brejo (Costus spicatus (Jacq.) SW.), melissa (Lippia alba N.E. Brown) e guaco (Mikania glomerata S.) com 4 coletas cada uma (Quadro 1).

Muitas dessas espécies também são citadas em trabalhos de natureza etnobotânica, embora não se apresentem na mesma ordem e quantidades, como observado nos estudos realizados por Silva et al. (2005), Calábria et al. (2008) e Kffuri (2008). 
Quadro 1. Mapeamento Etnobotânico da área de atendimento da UBS Vila Nova. São Lourenço, MG, 2018. F: família

\begin{tabular}{|c|c|c|c|c|}
\hline $\mathbf{N}^{\circ}$ Coleta & Nome Popular & Nome Científico & Família & Finalidade Terapêutica \\
\hline $\begin{array}{l}\text { F1-01; F3-01; F5-09; F18-01; } \\
\text { F25-02; F32-04; F40-01 }\end{array}$ & Tanchagem & Plantago major $\mathrm{L}$. & Plantaginaceae & $\begin{array}{c}\text { Gripes, inflamações, resfriados } \\
\text { e diarréia }\end{array}$ \\
\hline F1-02; F5-08; F23-02 & Poejo & Mentha pulegium L. & Lamiaceae & Gripes, resfriados e tosse \\
\hline $\begin{array}{l}\text { F1-03; F3-02; F4-08; F5-03; } \\
\text { F13-03; F19-03; F28-04; F31- } \\
\text { 03; F34-02; F36-04; F37-08; } \\
\text { F48-01; }\end{array}$ & Balsamo & Sedum dendroideum Moc & Crossulaceae & $\begin{array}{l}\text { Dores de estômago e úlceras, } \\
\text { azia, }\end{array}$ \\
\hline F1-04; F30-03 & Amora & Morus nigra $\mathrm{L}$. & Moraceae & Menopausa \\
\hline F1-05; F29-04; F34-03; F38-05; & Melissa & Lippia alba N. E. Brown & Verbenaceae & $\begin{array}{c}\text { Cólicas menstruais, cólicas e } \\
\text { calmante }\end{array}$ \\
\hline F1-06; F17-03; F24-02; F26-02 & Alfavaca & Ocimum selloi Benth. & Lamiaceae & Resfriados, gripes \\
\hline $\begin{array}{c}\text { F1-07; F5-04; F7-01; F8-02; } \\
\text { F9-02; F11-01; F13-02; F23- } \\
\text { 01; F28-03; F29-01; F33-02; } \\
\text { F42-03 }\end{array}$ & Erva cidreira & Melissa officinalis L. & Lamiaceae & Calmante e sonífero \\
\hline $\begin{array}{l}\text { F1-08; F2-05; F4-06; F7-03; } \\
\text { F14-03; F16-01; F18-03; F19- } \\
\text { 01; F26-01; F29-02; F35-02; } \\
\text { F37-02; F41-01; F43-02 }\end{array}$ & Alecrim & Rosmarinus officinalis $\mathrm{L}$. & Lamiaceae & $\begin{array}{c}\text { Diurético, temperos, calmante, } \\
\text { hábito }\end{array}$ \\
\hline F2-01; & Rosa branca & Rosa alba L. & Rasaceae & Inflamação nos olhos \\
\hline $\begin{array}{c}\text { F2-02; F9-04 F33-02; F34-04; } \\
\text { F36-02; }\end{array}$ & Manjericão & Ocimum basillicum $\mathrm{L}$. & Lamiaceae & $\begin{array}{c}\text { Calmante, ansiolítico, tempero, } \\
\text { hábito }\end{array}$ \\
\hline $\begin{array}{c}\text { F2-03; F4-05;F8-05; F21-01; } \\
\text { F40-02; F46-01 }\end{array}$ & Boldo brasileiro & Plectranthus barbatus A. & Lamiaceae & $\begin{array}{c}\text { Protetor do fígado, má } \\
\text { digestão, dor de estômago }\end{array}$ \\
\hline $\begin{array}{c}\text { F2-04; F3-06; F4-02; F5-02; } \\
\text { F31-01; F37-07 }\end{array}$ & Arruda & Ruta graveolens $\mathrm{L}$. & Rutaceae & Banhos, dores \\
\hline F2-06 & Guiné & Petiveria alliaceae $\mathrm{L}$. & Phitolacaceae & Sistema imunológico e gripes \\
\hline F2-07 & Quebra-pedra & Phyllanthus niruri L. & Euphorbiaceae & Pedra nos rins \\
\hline F2-08; F4-07; F37-05 & Romã & Punica granatum $\mathrm{L}$. & Punicaceae & Artrite, dor de garganta \\
\hline F3-03 & Laranjeira & Citrus sinensis $\mathrm{L}$. & Rutaceae & Ansiedade e depressão \\
\hline F3-04 & Goiabeira & Psidium guajava L. & Myrtaceae & Diarreia \\
\hline $\begin{array}{c}\text { F3-05; F4-01; F6-02; F7-02; } \\
\text { F8-01; F9-01; F10-05; F11-05; } \\
\text { F12-01; F13-01; F14-02; F15- } \\
\text { 01; F16-02; F18-02; F22-01; } \\
\text { F24-01; F27-04; F28-02; F29- } \\
\text { 03; F29-04; F31-02; F32-03; } \\
\text { F33-01; F34-01; F34-03; F36- } \\
\text { 03; F38-05; F41-02; F42-01; } \\
\text { F47-01; F49-01; }\end{array}$ & Hortelã & Mentha $\times$ villosa Huds & Lamiaceae & $\begin{array}{l}\text { Digestivo, contra gases, } \\
\text { calmante, hábito, azia }\end{array}$ \\
\hline $\begin{array}{l}\text { F3-07; F4-04; F6-01; F8-04; } \\
\text { F20-01; F22-03; F27-02; F38- } \\
\text { 03; F41-03; F43-01; F47-02 }\end{array}$ & Capim limão & Cymbopogon citratus & Poaceae & $\begin{array}{c}\text { Calmante, antidepressivo, } \\
\text { ansiedade }\end{array}$ \\
\hline $\begin{array}{c}\text { F3-08; F9-03; F11-03; F17-02; } \\
\text { F27-01 }\end{array}$ & Salsinha & Petroselinum crispum Mill. & Apiaceae & Inflamações, circulação, hábito \\
\hline $\begin{array}{c}\text { F4-03; F10-04; F20-03; F32-01; } \\
\text { F37-04, F39-01 }\end{array}$ & Funcho & Foeniculum vulgare Mill. & Apiaceae & $\begin{array}{l}\text { Digestivo, resfriados, } \\
\text { ansiedade, gases }\end{array}$ \\
\hline F5-01; F50-02 & Macelinha & Achyrocline satureioides DC. & Asteraceae & Azia e dores estomacais \\
\hline F5-05; F8-03; F19-02; F42-02 & Guaco & Mikania glomerata S. & Asteraceae & Tosse, bronquite e gripe \\
\hline F5-06; F23-03; F28-01 & Hera terrestre & Glechoma hederaceae $\mathrm{L}$. & Lamiaceae & Dor de garganta, tosse \\
\hline F5-07; F10-01, F27-03; F36-01 & Caninha do brejo & Costus spicatus (Jacq) SW. & Costaceae & Diurética, pressão arterial \\
\hline F5-10; & Levante & Mentha viridis $(\mathrm{L}) \mathrm{L}$. & Lamiaceae & Problemas respiratórios \\
\hline
\end{tabular}




\begin{tabular}{|c|c|c|c|c|}
\hline $\mathbf{N}^{\circ}$ Coleta & Nome Popular & Nome Científico & Família & Finalidade Terapêutica \\
\hline F10-02; F11-06; F22-02 & Ora-pro-nóbis & Pereskia aculeata Mill. & Cactaceae & Anemia, vitalidade \\
\hline F10-03; F11-08; F38-04 & Saião & Kalanchoe brasiliensis C. & Crassulaceae & Diabetes \\
\hline F11-02; F50-01 & Salvia & Salvia officinalis $\mathrm{L}$. & Lamiaceae & Cólicas, dor de cabeça, \\
\hline F11-04 & Terramicina & Alternanthera brasiliana $\mathrm{K}$. & Amaranthaceae & Antibiótico e cicatrizante \\
\hline F11-07 & Limoeiro & Citrus sp. & Rutaceae & Problemas digestivos \\
\hline F14-01; F25-01; F37-03 & Erva doce & Pimpinella anisium $\mathrm{L}$. & Apiaceae & Digestivo \\
\hline $\begin{array}{c}\text { F15-02; F35-01; F38-02; F44- } \\
\text { 01; F45-01; }\end{array}$ & Babosa & Aloe vera $(\mathrm{L})$ Burm. f. & Liliaceae & $\begin{array}{c}\text { Queimadura, cicatrizante, } \\
\text { queda de cabelo }\end{array}$ \\
\hline F16-03; & Carquejo & Baccharis trimera (Less) DC & Asteraceae & Diarréia e má digestão \\
\hline F17-01; F28-05 & Canfora & Artemisia camphorata Vill. & Asteraceae & Reumatismo e calmante \\
\hline F29-05 & Maracujá & Passiflora alata Curtis & Passifloraceae & Calmante \\
\hline F30-01 & Avocado & Persea americana Mill. & Lauraceae & Auxilio na perda de peso \\
\hline F30-02 & Acerola & Malpighia emarginata DC. & Malphigiaceae & Auxílio na perda de peso \\
\hline F30-04; F40-03 & Pitanga & Eugenia uniflora L. & Myrtaceae & Diabetes \\
\hline F30-05 & Hibisco & Hibiscus rosa-sinensis L. & Malvaceae & Auxilio na perda de peso \\
\hline F37-01 & Serralha & Sonchus oleraceus L. & Asteraceae & Inflamações \\
\hline F37-06 & Louro & Laurus nobilis L. & Lauraceae & Digestivo \\
\hline F38-01 & Aveloz & Euphorbia tirucalli $\mathrm{L}$. & Euphorbiaceae & Verruga \\
\hline F38-06 & Erva de São João & Hipericum perfotatum L. & Hypericaceae & Ansiedade e insônia \\
\hline F25-03 & Losna & Artemisia absinthium L. & Asteraceae & Vermes \\
\hline F20-02 & Boldo chinês & Plectranthus ornatos Codd. & Lamiaceae & Fígado e dores de estômago \\
\hline
\end{tabular}

Partes usadas do vegetal

A maior utilização das folhas também parece estar relacionada a uma coleta menos trabalhosa e maior disponibilidade desta parte do vegetal no decorrer do ano como apontam Castellucci et al. (2000). Jacoby et al. (2009), Dos Santos et al. (2008) e Costa e Mayworm (2011) também constataram em seus trabalhos que as folhas foram as partes mais utilizadas pelas pessoas para a utilização de seus preparos caseiros.

O modo de preparo caseiro que é mais utilizado, segundo os dados coletados em campo, são a infusão e decocção, sendo englobados como chás, para facilitar o entendimento deste tópico por parte da população.

A maceração, principalmente para o uso do boldo (Plectrathus barbatus A.), também se apresentou bastante utilizada pelos entrevistados. Segundo Lorenzi e Matos (2008) essa variação do preparo depende se a planta é preparada in natura ou na forma de droga vegetal (seca, estabilizada e acondicionada.

Ainda houve a alternativa "outras" se referindo basicamente, pelos relatos da população, da ingestão in natura crua e na forma de sucos, como foi o caso do balsamo (Sedum dendroideum Moc.) e da ora-pro-nóbis (Pereskia aculeata Mill.), respectivamente.

\section{Mapeamento etnobotânico}

No Quadro 1 observa-se as plantas medicinais coletadas e catalogadas, acrescidas das finalidades terapêuticas utilizadas pela população. No mapa (Figura 1) as coletas foram marcadas de acordo com a micro-área em que o agente comunitário de saúde atuava e com a ordem de coleta da planta dentro dessa microrregião. As cores das ruas indicam a micro-área onde foi realizada a coleta e as siglas são referentes ao número da coleta das 50 famílias, portanto, da sigla F1 à F50 destacadas com pontos na cor laranja. 
Figura 1. Mapa de atendimento da UBS Vila Nova e suas respectivas micro-áreas com locais de coleta do material botânico. São Lourenço, MG. 2018

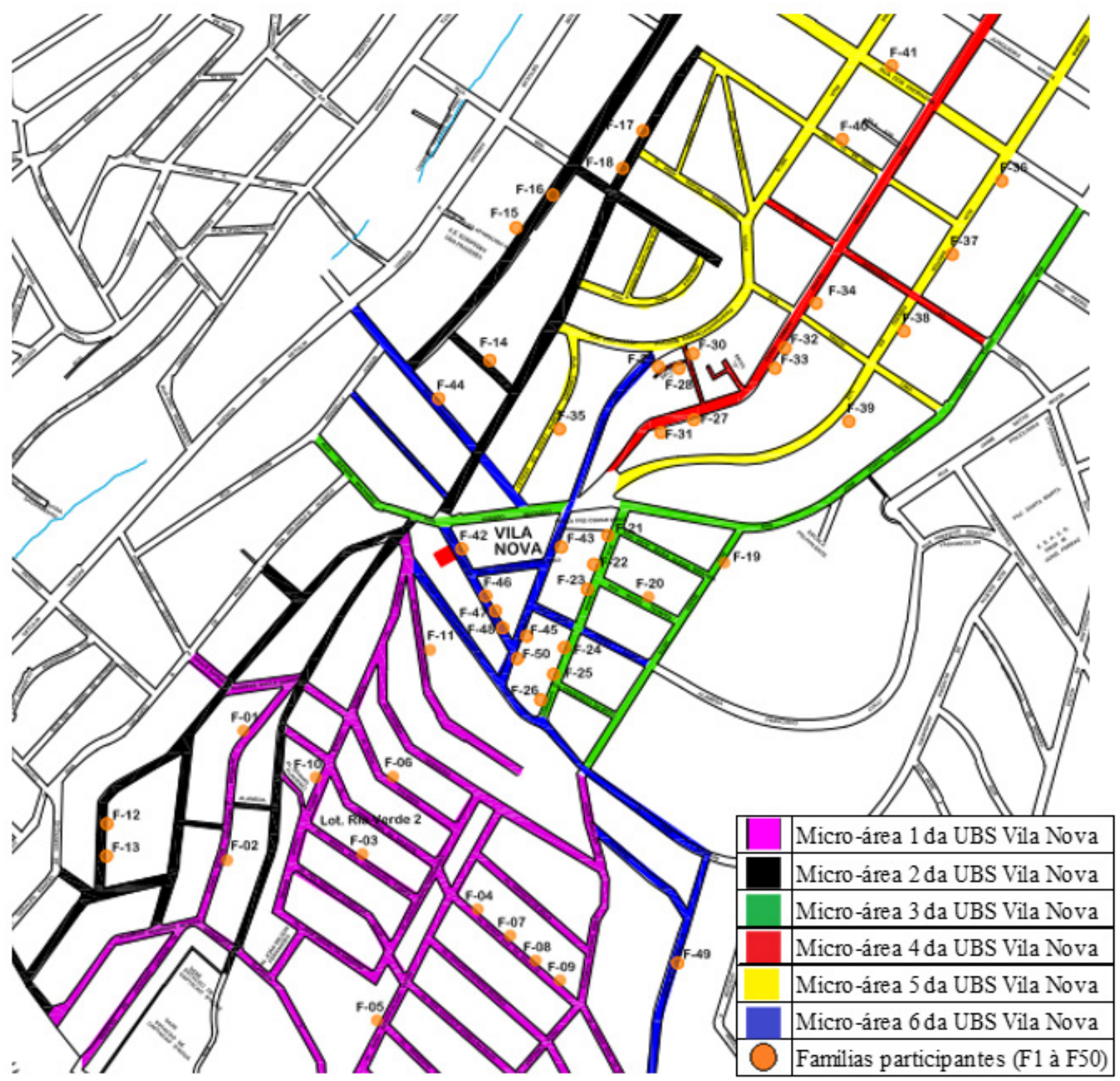

Fonte: dados da pesquisa, 2018.

Quanto à finalidade terapêutica, os participantes responderam de forma livre, sendo tomada nota e catalogado os usos apontados por elas. Destaca-se, curiosamente, que muitas pessoas relataram consumir o vegetal por hábito e sem uma finalidade terapêutica específica e outras cultivam para utilização como temperos (sentido paladar). Esse dado pode estar relacionado com práticas tradicionais, passadas de geração em geração, nas quais ficam o hábito, sem necessariamente uma explicação ou justificativa para consumo da planta.

Outro dado revelou a relação do cultivo comum das plantas pelas matriarcas. Para esse tratamento, destacaramse a hortelã (Mentha x villosa Huds.) e o funcho (Foeniculum vulgare Mil.). Na literatura, segundo Cunha et al. (2003), a hortelã tem ações que melhoram a digestão e aumentam o apetite, por possuírem poder de estimular principalmente a secreção do suco gástrico.

Dados da pesquisa de campo também indicaram que os problemas mais frequentes para os quais os entrevistados recorrem ao auxílio das plantas medicinais são gripes e resfriados, dores de barriga e problemas do trato digestivo em geral como gases e diarreias, bem como hipertensão, ansiedade e depressão, problemas do trato renal, cólicas menstruais e inflamações. Na literatura, Gély e Amorozo (1988), Hanazaki et al. (1996) e Silva-Almeida (1998) também observaram resultados semelhantes em seus estudos, porém em valores e nomenclaturas diferentes dos apresentados neste trabalho, pois essa alternativa era aberta à livre resposta dos entrevistados. 


\section{Conclusão}

Os dados da pesquisa de campo revelaram que as plantas medicinais para fins terapêuticos, ou por hábito de consumo, são muito utilizadas pela população cadastrada no Programa Saúde da Família na UBS do bairro Vila Nova na cidade de São Lourenço. Sendo que a maioria dos usuários cultiva suas plantas devido a suas origens rurais e seu conhecimento empírico passado por seus ancestrais.

Percebe-se ainda a pouca interação entre a política pública - Projeto Farmácia Verde - e as práticas com as plantas medicinais realizadas pela população.

As plantas são utilizadas de formas diversas, destacando-se o papel das mulheres em seu cultivo e conhecimento aplicado. Esses, nem sempre se conectam ao Projeto Farmácia Verde, ainda que a população reconheça em algum nível, a existência e importância do mesmo. Dessa maneira, analisa-se que os dados resultantes dessa pesquisa ajudam identificar possíveis lacunas na integração entre a população usuária e o Projeto Farmácia Verde, as quais devem ser olhadas com atenção, para o sucesso da política pública.

O levantamento etnobotânico realizado no trabalho de campo atesta a importância e expressão das plantas medicinais para a população local, além do seu alto grau de conhecimento sobre cultivo, uso e benefícios para saúde. Reiterando a importância social, econômica e cultural da Política Nacional de Práticas Integrativas e Complementares de Saúde (PNIPIC - SUS). Contundo, destaca-se também os desafios para o pleno conhecimento e usufruto dessa política pela população. A análise feita é a de que um reconhecimento mútuo (projeto - população), buscando valores em comum, identidades, ações e objetivos devem ser construídos e reconstruídos continuamente de forma colaborativa e participativa para o sucesso de todos.

\section{REFERÊNCIAS}

AGÊNCIA NACIONAL DE VIGILÂNCIA SANITÁRIA (ANVISA). Resolução da diretoria colegiada - RDC No 18, DE 03 DE ABRIL DE 2013

Albuquerque UP, Cruz da Cunha LVF, Lucena RFP, Alves RRN. 2014. Methods and techniques used to collect ethnobiological data. In: Methods and techniques in Ethnobiology and Ethnoecology. Humana Press, New York, NY. p. 15-37.

Amorozo MC de M, Reis MS, Ferri PH. 1996. A Abordagem etnobotânica na pesquisa de plantas medicinais. In: Di Stasi LC (Org.). Plantas medicinais: arte e ciência - um guia de estudo interdisciplinar. São Paulo: Editora da Universidade Estadual Paulista. p. 47-68.

Amorozo MCdeM. 2002. Uso e diversidade de plantas medicinais em Santo Antônio do Leverger, MT, Brasil. Acta Botânica Brasilica, 16(2):189-203.

Brasil, Ministério da Saúde. Secretaria Executiva. Secretaria de Atenção à Saúde. Secretaria de Ciência, Tecnologia e Insumos Estratégicos. . 2006. Política Nacional de Práticas Integrativas e Complementares no SUS. Brasília, DF, 92 p.

Brasil, Ministério da Saúde. Secretaria de Atenção à Saúde. Departamento de Atenção Básica. 2012. Práticas integrativas e complementares: plantas medicinais e fitoterapia na Atenção Básica/Ministério da Saúde. Secretaria de Atenção à Saúde. Departamento de Atenção Básica. - Brasília, DF.

Brasil, Ministério da Saúde. Secretaria de Atenção à Saúde. Departamento de Atenção Básica. 2015. Política nacional de práticas integrativas e complementares no SUS: atitude de ampliação de acesso. Secretaria de Atenção à Saúde. Departamento de Atenção Básica, 2. ed. Brasília, DF, 96 p. 
Brasil, Ministério da Saúde. Resolução no 1885, DE 27 DE MAIO DE 2009 da Secretaria de Saúde do Estado de Minas Gerais.

Calábria L, Cuba GT, Hwang SM, Marra JCF, Mendonça MF, Nascimento RC, Oliveira MR, Porto JPM, Santos DF, Silva BL, Soares TF, Xavier EM, Damasceno AA, Milani JF, Rezende CHA, Barbosa AAA, Canabrava HAN. 2008. Levantamento etnobotânico e etnofarmacológico de plantas medicinais em Indianópolis, Minas Gerais, Brasil. Revista Brasileira de Plantas Medicinais, 10(1):49-63.

Castellucci S, Lima MIS, Nordi N,Marques JGW. 2000. Plantas medicinais relatadas pela comunidade residente na Estação Ecológica de Jataí, município de Luís Antônio - SP: uma abordagem etnobotânica. Revista Brasileira de Plantas Medicinais, 3(1):51-60.

Costa VP, Mayworm MAS. 2011. Plantas medicinais utilizadas pela comunidade do bairro dos Tenentes-município de Extrema, MG, Brasil. Revista Brasileira de Plantas Medicinais, 13(3):282-292.

Cunha APda, Silva APda, Roque OR. 2003. Plantas e produtos vegetais em fitoterapia. Lisboa: Fundação Calouste Gulbenkian.

De Mello Botelho J, Do Nascimento LFAP, Lamano FM. 2014. Prática de cultivo e uso de plantas domésticas em diferentes cidades brasileiras. Ciência Rural, 44(10):1810-1815.

De Oliveira FC, Albuquerque UPde, Fonseca-Kruel VSda, Hanazaki N. 2009. Avanços nas pesquisas etnobotânicas no Brasil. Acta Botanica Brasilica, 23:590-605.

Dos Santos M, De Lima M.R, Ferreira M.dasGR. 2008. Uso de plantas medicinais pela população de Ariquemes, em Rondônia. Horticultura Brasileira, 26(2):244-250.

Gély A, Amorozo MCdeM. 1988. Uso de plantas medicinais por caboclos do baixo Amazonas Barcarena, PA, Brasil. Boletim Museu Paraense Emilio Goedi, Sér. Bot 4(1).

Hanazaki N, De Freitas Leitao-Filho H, Begossi A. 1996. Uso de recursos na Mata Atlântica: o caso da Ponta do Almada (Ubatuba, Brasil). Interciencia, 21(6):268-276.

IBGE. 2013. Instituto Brasileiro de Geografia e Estatística. Censo Demográfico. Minas Gerais.

IBGE. 2015. Instituto Brasileiro de Geografia e Estatística. Censo Demográfico. Minas Gerais.

Jacoby C, Coltro EM, Sloma D C, Muller J, Dias LA, Luft M, Neto RM. 2009. Plantas medicinais utilizadas pela comunidade rural de Guamirim, Município de Irati, PR. RECEN-Revista Ciências Exatas e Naturais, 4(1):79-89.

Kffuri CW. 2008. Etnobotânica de plantas medicinais no município de Senador Firmino, Minas Gerais. 2008. f. 88. Tese de Doutorado. Universidade Federal de Viçosa, Viçosa.

Lins J. 2009. A cidadania e a prática de saúde da família. In: Pinheiro R, Martins PH. Avaliação em saúde na perspectiva do usuário: abordagem multicêntrica. Rio de Janeiro: Cepesc, 2009.

Lorenzi H, Matos FJ. 2008. Plantas Medicinais no Brasil: nativas e exóticas. 2. ed. Nova Odessa, SP: Instituto Plantarum. Martin, GJ. 1995. Ethnobotany, a methods manual. Ed. Chapman \& Hall, Londres: 268 p. 
Silva-Almeida MF, Amorozo MCM. 1998. Medicina popular no distrito de Ferraz, município de Rio Claro, Estado de São Paulo. Brazilian Journal of Ecology, 2(2):36-46.

Silva FS, MACEDO RLG, VENTURIM N, MORAIS VdeM, GOMES, JE. 2005. Levantamento etnobotânico das plantas medicinais da zona rural do município de Piumhi, Minas Gerais. Revista Científica Eletrônica de Engenharia Florestal, $3(6): 1-4$.

Sousa IMC, Tesser CD, Hortale V. 2012. Práticas integrativas e complementares: oferta e produção de atendimentos no SUS e em municípios selecionados. Caderno de Saúde Pública, 28(11):2143-2154.

Souza C. 2006. Políticas públicas: uma revisão da literatura. Sociologias, (16):20-45.

Trindade C, Sartório M, Rezende P. 2008. Farmácia Viva: utilização de plantas medicinais. Viçosa: CPT. 246p. 